\title{
LIE GROUPS ASSOCIATED WITH PSEUDO GROUPS ${ }^{1}$
}

\author{
H. H. JOHNSON
}

The transformations of a pseudo group which leave a given point invariant induce a group of transformations on the space of $k$-jets with that point as source and target. This group is a subgroup of a Lie group. When the pseudo group contains all translations, this group, for $k$ sufficiently large, uniquely determines the pseudo group, at least under smoothness assumptions common in the older literature.

1. Definitions. All functions, manifolds, etc., are assumed real analytic. "Transformation" means analytic homeomorphism. Let $G$ be a pseudo group [5] of local transformations on a neighborhood $Q$ of the origin 0 in $E^{n}$, Euclidean $n$-space. If $f$ is in $G, U(f)$ and $V(f)$ denote the domain and range of $f$, respectively. Let $G$ be the set of all elements of $G$ containing 0 in their domain which leave 0 invariant.

$J^{k}(Q)$ denotes the set of all invertible $k$-jets $j_{x}^{k}(f)$ of $Q$ into itself [1]. It has coordinates $\left(x^{i}, y^{i}, p_{j_{1}}^{i}, \cdots, p_{f_{1}}^{i} \ldots j_{k}\right)$, where $\left(x^{i}\right)$ are those of the source $x,\left(y^{i}\right)$ those of the target $f(x)$, and

$$
p_{j_{1} \cdots j_{h}}^{i}=\left(\partial^{h} y^{i}\right) /\left(\partial x^{j_{1}} \cdots \partial x^{j_{h}}\right)(x) .
$$

$\alpha$ and $\beta$ denote the source and target projections, as usual. If $g$ is in $G$, let $p^{k}(g)\left(j_{x}^{k}(f)\right)=j_{x}^{k}(g \circ f)$ for all $j_{x}^{k}(f)$ in $\beta^{-1}(U(g))$.

Let $B^{k}$ be the set of all $j_{0}^{k}(f)$ where $f(0)=0$. $B^{k}$ is a Lie group under the natural product $j_{0}^{k}\left(f_{1}\right) \cdot j_{0}^{k}\left(f_{2}\right)=j_{0}^{k}\left(f_{1} \circ f_{2}\right)$. Let $G^{k}$ be the set of elements in $B^{k}$ coming from $G$.

Investigating relations between $G^{k}$ and $G$ is the main concern of this paper. Conditions will be imposed on $G$ of two types. The first is special and imposes a restriction on the types of pseudo groups studied. The rest are regularity conditions and seem to have been assumed or proved for the pseudo groups studied by S. Lie and E. Cartan.

2. $G^{k}$ and $G$. Condition (A): $G$ contains all translations, i.e., given any two points $y_{1}$ and $y_{2}$ in $Q$, there exists a translation in $G$ defined on a neighborhood of $y_{1}$ which carries $y_{1}$ into $y_{2}$.

Proposition. If $G$ satisfies (A) then $G$ completely determines $G$.

Proof. Let $t_{y}$ denote a translation in $G$ carrying $y$ to 0 . If $f$ is in

Received by the editors January $5,1962$.

${ }^{1}$ Supported by ONR Contract 202736. 
$G, y$ in $U(f)$, then $t_{f(v)}^{-1} \circ f \circ t_{y}$ is in $G$, so the pseudo group generated by all elements $t_{\nu_{1}}^{-1} \circ G \circ t_{y_{2}}$ generates $G$.

There is a natural analytic homeomorphism $e: Q \times Q \times B^{k} \rightarrow J^{k}(Q)$ defined by $e\left(x_{1}, y_{1}, j_{0}^{k}(f)\right)=j_{x_{1}}^{k}\left(t_{y_{1}}^{-1} \circ f \circ t_{x_{1}}\right)$. In coordinates, $e\left(x_{1}^{i}, y_{1}^{i}, 0,0, p_{j_{1}}^{i}, \cdots, p_{p_{1}}^{i} \cdots j_{k}\right)=\left(x_{1}^{i}, y_{1}^{i}, p_{j_{1}}^{i}, \cdots, p_{j_{1}}^{i} \cdots j_{k}\right)$. The statement that a function $F$ on $J^{k}(Q)$ is independent of $\alpha\left(J^{k}(Q)\right)$ or $\beta\left(J^{k}(Q)\right)$ will mean $F \circ e$ is independent of the first or second $Q$ in $Q \times Q \times B^{k}$, respectively.

Condition (B): For some $k$ there exist independent real-valued functions $Y^{1}, \cdots, Y^{r}$ on $J^{k}(Q)$ such that $f$ belongs to $G$ if and only if $Y^{r} \circ p^{k}(f)=Y^{r}, r=1, \cdots, r$. Since any function depending only on $\alpha\left(J^{k}(Q)\right)$ is trivially invariant, it is assumed that the $Y^{r}$ are indepent of $\alpha\left(J^{k}(Q)\right)$.

This regularity condition, which asserts that $G$ is defined by "differential invariants," was proved by S. Lie for his transformation groups [2]. In some examples the $Y^{r}$ may not be defined or independent except on some open subset $U$ of $J^{k}(Q)$ where $\beta(U)=Q$. The arguments should be modified in this case.

Proposition. If $G$ satisfies (A) and (B) the functions $Y^{r}$ are independent of $\beta\left(J^{k}(Q)\right), r=1, \cdots, r$.

PROOF. Yr $\operatorname{Yr}^{r} e\left(x_{1}, y_{1}, j_{0}^{k}(f)\right)=\operatorname{Yr}^{r}\left(j_{x_{1}}^{k}\left(t_{y_{1}}^{-1} \circ f \circ t_{x_{1}}\right)\right)=Y^{r} \circ p^{k}\left(t_{y_{1}}^{-1}\right)$ $\cdot\left(j_{x_{1}}^{k}\left(f \circ t_{x_{1}}\right)\right)=Y^{r}\left(j_{x_{1}}^{k}\left(f \circ t_{x_{1}}\right)\right)$.

Let $q$ be the projection of $Q \times Q \times B^{k}$ onto $B^{k}$. If (A) and (B) are satisfied there exist natural real-valued functions $Z^{r}$ on $B^{k}$ such that $Y^{r} \circ e=Z^{r} \circ q, r=1, \cdots, r$. By definition $G^{k}$ acting on $B^{k}$ by leftmultiplication leaves each $Z^{r}$ invariant.

Under condition (B), $G$ is defined by $Y^{1}, \cdots, Y^{r}$. These $Y^{r}$ define a system of partial differential equations which all elements of $G$ must satisfy. The remaining conditions are regularity conditions on these equations.

In [5] it is shown that there exist on $J^{k}(Q)$ 1-forms $\phi^{i}, \phi_{j_{1}}^{i} \ldots j_{h}$; $i, j_{1}, \cdots, j_{h}=1, \cdots, n ; h=1, \cdots, k-1$, such that a transformation $F: J^{k}(Q) \rightarrow J^{k}(Q)$ is locally a restriction of some $p^{k}(f)$ for $f: Q \rightarrow Q$ if and only if $F^{*}$ leaves each of these 1-forms invariant and $\alpha \circ F=\alpha$. (In [5] $p^{k}(f)$ is defined to act on $j_{x}^{k}(g)$ by $j_{x}^{k}\left(g \circ f^{-1}\right)$, so the theory there is dual to that used here.)

Let $\rho_{1}$ and $\rho_{2}$ denote the projections of $J^{k}(Q) \times J^{k}(Q)$ onto the first and second factors, respectively. Consider on $J^{k}(Q) \times J^{k}(Q)$ the exterior differential system $\Sigma(Y)$ generated by

$$
\begin{gathered}
Y^{r} \circ \rho_{1}-Y^{r} \circ \rho_{2}, \quad \alpha \circ \rho_{1}-\alpha \circ \rho_{2}, \\
\rho_{1}^{*} \phi^{i}-\rho_{2}^{*} \phi^{i}, \quad \rho_{1}^{*} \phi_{j_{1}}^{i} \cdots j_{h}-\rho_{2}^{*} \phi_{j_{1}}^{i} \cdots j_{h},
\end{gathered}
$$


$r=1, \cdots, r ; i, j_{1}, \cdots, j_{h}=1, \cdots, n ; h=1, \cdots, k-1$, and their exterior derivatives. The second factor of $J^{k}(Q) \times J^{k}(Q)$ will be taken as independent variables. Then any solution $\psi$ has the form $\psi\left(j_{x}^{k}(f)\right)$ $=\left(p^{k}(g)\left(j_{x}^{k}(f)\right), j_{x}^{k}(f)\right)$, where $g$ is in $G$.

Condition $(\mathrm{C}): \Sigma(Y)$ is involutive at all integral points.

Proposition. If (A), (B), and (C) are all satisfied, then $Z^{1}, \cdots, Z^{r}$ constitute a full set of invariants for $G^{k}$. That is, if $Z$ is any invariant real-valued function on $B^{k}$ it must be locally a function of $Z^{1}, \cdots, Z^{r}$.

Proof. If $j_{0}^{k}\left(f_{1}\right)$ and $j_{0}^{k}\left(f_{2}\right)$ belong to $B^{k}$ and satisfy $Z^{r}\left(j_{0}^{k}\left(f_{1}\right)\right)$ $=Z^{r}\left(j_{0}^{k}\left(f_{2}\right)\right), r=1, \cdots, r$, then $\left(j_{0}^{k}\left(f_{1}\right), j_{0}^{k}\left(f_{2}\right)\right)$ is an integral point of $\Sigma(Y)$. Hence there exists $g$ in $G$ such that $p^{k}(g)\left(j_{0}^{k}\left(f_{2}\right)\right)=j_{0}^{k}\left(f_{1}\right)$. Since $g(0)=g\left(f_{2}(0)\right)=f_{1}(0)=0, g$ is in $G$, and hence $j_{0}^{k}(g)$ is in $G^{k}$. It follows that $Z\left(j_{0}^{k}\left(f_{1}\right)\right)=Z\left(j_{0}^{k}\left(f_{2}\right)\right)$ whenever $Z^{r}\left(j_{0}^{k}\left(f_{1}\right)\right)=Z^{r}\left(j_{0}^{k}\left(f_{2}\right)\right), r=1, \cdots, r$.

From this one may say that $G^{k}$ determines $G$ uniquely, since its invariant functions determine the differential invariants of $G$. In conclusion a condition will be given under which $G^{k}$ is characterized by this property, i.e., $G^{k}$ contains all transformations in $B^{k}$ which leave $Z^{1}, \cdots, Z^{r}$ invariant under left-multiplication.

Suppose $j_{0}^{k}(g)$ is in $B^{k}$ and leaves each $Z^{1}, \cdots, Z^{r}$ invariant. The manifold

$$
V\left(j_{0}^{k}(g)\right)=\left\{\left(j_{0}^{k}(g \circ f), j_{0}^{k}(g)\right) \mid j_{0}^{k}(f) \text { is in } B^{k}\right\}
$$

is an integral submanifold of $\Sigma(Y)$. If one can find an integral of $\Sigma(Y)$ of maximal dimension containing $V\left(j_{0}^{*}(g)\right)$, it would arise from some $p^{k}(\xi)$ where $g$ is in $G$. Then $j_{0}^{k}(g)=j_{0}^{k}(\xi)$, so $j_{0}^{k}(g)$ would belong to $G^{k}$.

Condition (D): Every $V\left(j_{0}^{k}(g)\right)$, where $j_{0}^{k}(g)$ leaves $Z^{1}, \cdots, Z^{r}$ invariant, has its tangent space at each of its points contained in a regular flag of $\Sigma(Y)$. (For this terminology, see [3].)

Theorem. If $G$ satisfies (A) (B), (C), and (D), then $G^{k}$ consists in all transformations of the Lie group $B^{k}$ which leave invariant a finite number of real-valued functions and $G^{k}$ uniquely determines $G$.

\section{BIBLIOGRAPHY}

1. C. Ehresmann, Introduction d la theorie des structures infinitesimals et des pseudo groupes de Lie, Colloq. Internat. de Géométrie Différentielle du C.N.R.S., 1953.

2. S. Lie, Gesammelte Abhandlungen, Vol. 6, 2nd. ed., B. G. Teubner, Leipzig, 1922, p. 362.

3. M. Kuranishi, E. Cartan's prolongation theorem, Amer. J. Math. 79 (1957), 1-47.

4. - On the local theory of continuous infinite pseudo groups. I, Nagoya Math. J. 15 (1959), 225-260.

5. - On the local theory of continuous infinite pseudo groups. II, Nagoya Math. J. 19 (1961), 55-92.

UNIVERSITY OF WASHINGTON 